\title{
Quantum stochastic processes: boson and fermion Brownian motion*
}

\author{
A.E.Kobryn ${ }^{1,2}$, T.Hayashi ${ }^{1}$, T.Arimitsu ${ }^{1}$ \\ 1 Institute of Physics, University of Tsukuba, Ibaraki 305-8571, Japan \\ 2 Institute for Molecular Science, Myodaiji, Okazaki, Aichi 444-8585, Japan
}

Received September 5, 2003

Dynamics of quantum systems which are stochastically perturbed by linear coupling to the reservoir can be studied in terms of quantum stochastic differential equations (for example, quantum stochastic Liouville equation and quantum Langevin equation). In order to work it out one needs to define the quantum Brownian motion. As far as only its boson version has been known until recently, in the present paper we present the definition which makes it possible to consider the fermion Brownian motion as well.

Key words: stochastic processes, boson Brownian motion, fermion Brownian motion

PACS: 02.50.Ey, 05.30.-d

The description of time-dependent behavior of non-equilibrium systems involving stochastic forces can be given with the help of the Langevin equation. It is the stochastic differential equation for dynamic variables and is of fundamental importance in the theory of Brownian motion $[1,2]$. Random forces in Langevin equation are usually described by Gaussian white stochastic processes [3] since such a description is a convenient model for the processes with short correlation times. Similarly, from the mathematical point of view, white noise can be considered to be a limit of some well specified stochastic processes. Stochastic integral with respect to such processes is defined as a kind of a Riemann-Stieltjes one [4] where multiplication between the stochastic increment and integrand is commonly considered in the form of Itô [5] or Stratonovich [6].

The Langevin equation can be used to calculate various time correlation functions. Now it is radically extended to solve numerous problems arising in different areas [7-11]. In particular, the theory of Brownian motion itself has been extended to the situations where the "Brownian particle" is not a real particle anymore, but instead it possesses some collective properties of a macroscopic system. A corresponding equation in the phase space or the Liouville space of quantum mechanical

*Invited paper. 
statistical operators can be also considered as a sort of stochastic differential equation. In order to investigate classical stochastic systems, the stochastic Liouville equation was first introduced by Anderson [12] and Kubo [13-15].

There were several attempts to extend the classical theory (both Langevin and stochastic Liouville equations) to the quantum cases. Study of the Langevin equation for quantum systems has its origin in papers by Senitzky [16], Schwinger [17], Haken [18-20] and Lax [21], where they investigated a quantum mechanical damped harmonic oscillator in connection with laser systems. In particular, it was shown that the quantum noise, i.e. the spontaneous emission, can be treated in a way similar to the thermal fluctuations, and that the noise source has non-zero second moments proportional to a quantity which can be associated with a quantum analogue of a diffusion coefficient. As it was noticed by Kubo [22] in his discussion with van Kampen, the random force must be an operator defined in its own Hilbert space, which does not happen in the classical case since there is no consideration of space for the random force.

Mathematical study of the quantum stochastic processes was initiated by Davies $[23,24]$. Essential progress in this subject has been made by mathematicians [25-31]. For instance, quantum mechanical analogues of Wiener processes [25] and quantum Itô formula for boson systems [26,27] were defined first by Hudson and co-authors. The classical Brownian motion is replaced here by the pair of one-parameter unitary group automorphisms, namely by the boson random force annihilation and creation operators with time indices in the boson Fock space, named quantum Brownian motion. Fermion stochastic calculus was first suggested by Applebaum, Hudson and Parthasarathy [32-35]. In these papers, they developed the fermion analog of the corresponding boson theory [27] in which the annihilation and creation processes are fermion field operators in the fermion Fock space. Within the framework of this formalism, the Itô-Clifford integral [36] - fermion analog of the classical Brownian motion - is contained as a special case.

In this paper we give the definition of boson and fermion Brownian motion following the approach suggested by Hudson and Parthasarathy [27,34]. We do not pay much attention to purely mathematical aspects of the subject. Instead of that we try to represent the material in the terms which are easy for physicists to understand. We also consider quantum Brownian motion with allowance for thermal degree of freedom.

Let $\Gamma_{\mathrm{s}}^{0}$ denote the boson Fock space (the symmetric Fock space) over the Hilbert space $\mathcal{H}=L^{2}\left(\mathbb{R}_{+}\right)$of square integrable functions, and $b_{t}$ and $b_{t}^{\dagger}$ denote, respectively, boson annihilation and creation operators at time $t \in[0, \infty)$ satisfying the canonical commutation relations

$$
\left[b_{t}, b_{s}^{\dagger}\right]=\delta(t-s), \quad\left[b_{t}, b_{s}\right]=\left[b_{t}^{\dagger}, b_{s}^{\dagger}\right]=0 .
$$

The bra- and ket-vacuums ( $\mid$ and $\mid)$, respectively, are defined by $\left(\mid b_{t}^{\dagger}=0\right.$ and $\left.b_{t} \mid\right)=0$. The space $\Gamma_{\mathrm{s}}^{0}$ is equipped with a total family of exponential vectors

$$
\left(e(f)\left|=\left(\left|\exp \left\{\int_{0}^{\infty} \mathrm{d} t f^{*}(t) b_{t}\right\}, \quad\right| e(g)\right)=\exp \left\{\int_{0}^{\infty} \mathrm{d} t g(t) b_{t}^{\dagger}\right\}\right|\right),
$$


whose overlapping is $(e(f) \mid e(g))=\exp \left\{\int_{0}^{\infty} \mathrm{d} t f^{*}(t) g(t)\right\}$. Here $f, g \in \mathcal{H}$. The dense span of exponential vectors we denote by $\mathcal{E}$. Operators $b_{t}, b_{t}^{\dagger}$ and exponential vectors are characterized by the relations $\left(e(f) \mid b_{t}^{\dagger}=\left(e(f) \mid f^{*}(t)\right.\right.$ and $\left.\left.b_{t} \mid e(g)\right)=g(t) \mid e(g)\right)$.

Let us introduce operator $U_{t}$ defined as

$$
U_{t}=\sigma_{<} P_{[0, t]}+\sigma_{>} P_{(t, \infty)},
$$

where $\sigma_{<}$and $\sigma_{>}$are two independent parameters taking values \pm 1 , and $P_{[a, b]}(a \leqslant b)$ is an operator on $\mathcal{H}$ of multiplication by the indicator function whose action reads

$$
P_{[a, b]} \int_{0}^{\infty} \mathrm{d} t g(t)=\int_{0}^{\infty} \mathrm{d} t \theta(t-a) \theta(b-t) g(t) .
$$

Here, $\theta(t)$ is the step function specified as

$$
\theta(t)=\left\{\begin{array}{l}
1, \quad t \geqslant 0 \\
0, \quad t<0
\end{array}\right.
$$

Operator $P_{[a, b]}$ has the following properties:

$$
P_{[a, b]}^{2}=P_{[a, b]}, \quad P_{[a, b]}^{\dagger}=P_{[a, b]}, \quad P_{[a, b]} P_{[c, d]}=P_{[c, d]} P_{[a, b]},
$$

which are easily verified using definition (4). Then we see that operator $U_{t}$ is unitary, and satisfies

$$
U_{t}^{2}=I, \quad U_{t}^{\dagger}=U_{t}, \quad U_{t} U_{s}=U_{s} U_{t}
$$

where $I$ is the identity operator. The so-called reflection process $J_{t} \equiv J_{t}\left(U_{t}\right), t \in \mathbb{R}_{+}$, whose action on $\mathcal{E}$ is given by [34]

$$
\left.\left.\left.J_{t} \mid e(g)\right)=\mid e\left(U_{t} g\right)\right)=\exp \left\{U_{t} \int_{0}^{\infty} \mathrm{d} t^{\prime} g\left(t^{\prime}\right) b_{t^{\prime}}^{\dagger}\right\} \mid\right),
$$

inherits properties of the operator $U_{t}(7)$, i.e.

$$
J_{t}^{2}=I, \quad J_{t}^{\dagger}=J_{t}, \quad J_{t} J_{s}=J_{s} J_{t}
$$

and does not change the vacuum: $\left(\left|J_{t}=\left(\left|, J_{t}\right|\right)=\right|\right)$.

Let us now consider new operators

$$
\mathfrak{b}_{t}=J_{t} b_{t}, \quad \mathfrak{b}_{t}^{\dagger}=b_{t}^{\dagger} J_{t}
$$

Apparently, they annihilate vacuums: $\left(\left|\mathfrak{b}_{t}^{\dagger}=0, \mathfrak{b}_{t}\right|\right)=0$. The following matrix elements

$$
\begin{aligned}
& \left(e(f)\left|\left[J_{t}, b_{s}\right]_{-\sigma}\right| e(g)\right)=\left(e(f)\left|\left\{1-\sigma\left[\sigma_{>}+\left(\sigma_{<}-\sigma_{>}\right) \theta(t-s)\right]\right\} g(s) J_{t}\right| e(g)\right), \\
& \left(e(f)\left|\left[\mathfrak{b}_{t}, \mathfrak{b}_{s}^{\dagger}\right]_{-\sigma}\right| e(g)\right)=\left(e(f)\left|\left\{\delta(t-s)+J_{t} J_{s}\left(\sigma_{>} \sigma_{<}-\sigma\right) f^{*}(s) g(t)\right\}\right| e(g)\right),
\end{aligned}
$$


are valid for $f, g \in \mathcal{H}$, where for arbitrary operators $A$ and $B$ the (anti-)commutator is defined as

$$
[A, B]_{-\sigma}=A B-\sigma B A, \quad \sigma= \pm 1 .
$$

Then the requirement of equal-time (anti-)commutativity between $J_{t}$ and $b_{t}$, i.e. $\left[J_{t}, b_{t}\right]_{-\sigma}=0$, gives $1-\sigma \sigma_{<}=0$, while the requirement of canonical (anti-)commutation relation

$$
\left[\mathfrak{b}_{t}, \mathfrak{b}_{s}^{\dagger}\right]_{-\sigma}=\delta(t-s)
$$

leads to $\sigma_{>} \sigma_{<}-\sigma=0$. All those conditions are satisfied when $\sigma_{<}=\sigma$ and $\sigma_{>}=+1$. Then the operator $U_{t}$ turns out to be

$$
U_{t}=\sigma P_{[0, t]}+P_{(t, \infty)}
$$

Note that for a boson system, i.e. $\sigma=1, U_{t}=I$ and the operators $\mathfrak{b}_{t}$ and $\mathfrak{b}_{t}^{\dagger}$ reduce, respectively, to $b_{t}$ and $b_{t}^{\dagger}$.

We see that the generalized quantum Brownian motion, defined by

$$
\mathfrak{B}_{t}=\int_{0}^{t} \mathrm{~d} t^{\prime} \mathfrak{b}_{t^{\prime}}, \quad \mathfrak{B}_{t}^{\dagger}=\int_{0}^{t} \mathrm{~d} t^{\prime} \mathfrak{b}_{t^{\prime}}^{\dagger}
$$

with $\mathfrak{B}_{0}=0, \mathfrak{B}_{0}^{\dagger}=0$, satisfies $\left[\mathfrak{B}_{t}, \mathfrak{B}_{s}^{\dagger}\right]_{-\sigma}=\min (t, s)$. The case $\sigma=1$ represents the boson Brownian motion [27,30], whereas the case $\sigma=-1$ represents the fermion Brownian motion [34]. Their increments annihilate the vacuum, i.e.

$$
\begin{aligned}
\mathrm{d} \mathfrak{B}_{t} & =\mathfrak{B}_{t+\mathrm{d} t}-\mathfrak{B}_{t}=\mathfrak{b}_{t} \mathrm{~d} t, & \left.\mathrm{~d} \mathfrak{B}_{t} \mid\right)=0 \\
\mathrm{~d} \mathfrak{B}_{t}^{\dagger}=\mathfrak{B}_{t+\mathrm{d} t}^{\dagger}-\mathfrak{B}_{t}^{\dagger}=\mathfrak{b}_{t}^{\dagger} \mathrm{d} t, & & \left(\mid \mathrm{d} \mathfrak{B}_{t}^{\dagger}=0\right.
\end{aligned}
$$

and their matrix elements read

$$
\begin{array}{ll}
\left(e(f)\left|\mathrm{d} \mathfrak{B}_{t}\right| e(g)\right)=\left(e(f)\left|J_{t} g(t) \mathrm{d} t\right| e(g)\right), & \left(e(f)\left|\mathrm{d} \mathfrak{B}_{t} \mathrm{~d} \mathfrak{B}_{t}\right| e(g)\right)=0, \\
\left(e(f)\left|\mathrm{d} \mathfrak{B}_{t}^{\dagger}\right| e(g)\right)=\left(e(f)\left|f^{*}(t) \mathrm{d} t J_{t}\right| e(g)\right), & \left(e(f)\left|\mathrm{d} \mathfrak{B}_{t} \mathrm{~d} \mathfrak{B}_{t}^{\dagger}\right| e(g)\right)=\mathrm{d} t(e(f) \mid e(g)) .
\end{array}
$$

Here we neglected the terms of the order higher than $\mathrm{d} t$. The latter equations are summarized in the following table of multiplication rules for increments $\mathrm{d} \mathfrak{B}_{t}$ and $\mathrm{d} \mathfrak{B}_{t}^{\dagger}$ :

\begin{tabular}{l|lll} 
& $\mathrm{d} \mathfrak{B}_{t}$ & $\mathrm{~d} \mathfrak{B}_{t}^{\dagger}$ & $\mathrm{d} t$ \\
\hline $\mathrm{d} \mathfrak{B}_{t}$ & 0 & $\mathrm{~d} t$ & 0 \\
$\mathrm{~d} \mathfrak{B}_{t}^{\dagger}$ & 0 & 0 & 0 \\
$\mathrm{~d} t$ & 0 & 0 & 0
\end{tabular}

Quantum stochastic calculus with consideration for thermal degree of freedom can be derived within the framework of Non-Equilibrium Thermo Field Dynamics (NETFD). It is a unified formalism, which enables us to treat dissipative quantum 
systems by the method similar to the usual quantum mechanics and quantum field theory, which accommodates the concept of the dual structure in the interpretation of nature, i.e. in terms of the operator algebra and the representation space. Information about the general structure of NETFD can be found in many papers and we first of all refer to the original source [37] and to the review article [38]. With that in mind, we now consider a tensor product space $\hat{\Gamma}=\Gamma_{s}^{0} \otimes \tilde{\Gamma}_{s}^{0}$. Its vacuum states $\left.\left.\mid\right)\right)$ and exponential vectors $\mid e(f, g)))$ are defined through the "principle of correspondence" $[37]$

$$
\begin{aligned}
\mid)) & \longleftrightarrow \mid)(\mid, \\
\mid e(f, g))) & \longleftrightarrow \mid e(f))(e(g) \mid .
\end{aligned}
$$

Annihilation and creation operators acting on $\hat{\Gamma}$ are defined through

$$
\begin{aligned}
\left.\left.b_{t} \mid e(f, g)\right)\right) & \left.\longleftrightarrow b_{t} \mid e(f)\right)(e(g) \mid, & & \left.\left.\tilde{b}_{t} \mid e(f, g)\right)\right) \\
\left.\left.b_{t}^{\dagger} \mid e(f, g)\right)\right) & \left.\longleftrightarrow b_{t}^{\dagger} \mid e(f)\right)(e(g) \mid, & \left.\left.\tilde{b}_{t}^{\dagger} \mid e(f, g)\right)\right) & \longleftrightarrow \mid e(f))\left(e(g) \mid b_{t},\right.
\end{aligned}
$$

and similarly for $J_{t}$ and $\tilde{J}_{t}$, i.e.

$$
\left.\left.\left.\left.\left.J_{t} \mid e(f, g)\right)\right) \longleftrightarrow J_{t} \mid e(f)\right)\left(e(g)\left|, \quad \tilde{J}_{t}\right| e(f, g)\right)\right) \longleftrightarrow \mid e(f)\right)\left(e(g) \mid J_{t} .\right.
$$

Algebra of commutation relations between these operators reads

$$
\begin{array}{lll}
{\left[b_{t}, b_{s}^{\dagger}\right]=\left[\tilde{b}_{t}, \tilde{b}_{s}^{\dagger}\right]=\delta(t-s),} & & {\left[b_{t}, \tilde{b}_{s}\right]=\left[b_{t}, \tilde{b}_{s}^{\dagger}\right]=0} \\
{\left[J_{t}, \tilde{b}_{s}\right]=\left[\tilde{J}_{t}, b_{s}\right]=0,} & & {\left[J_{t}, b_{t}\right]_{-\sigma}=\left[\tilde{J}_{t}, \tilde{b}_{t}\right]_{-\sigma}=0 .}
\end{array}
$$

Let us now consider new operators defined by

$$
\begin{aligned}
\mathfrak{b}_{t} & =J_{t} b_{t}, & & \mathfrak{b}_{t}^{\dagger}=b_{t}^{\dagger} J_{t}, \\
\tilde{\mathfrak{b}}_{t} & =\hat{\tau} \tilde{J}_{t} \tilde{b}_{t}, & \tilde{\mathfrak{b}}_{t}^{\dagger} & =\hat{\tau} \tilde{b}_{t}^{\dagger} \tilde{J}_{t},
\end{aligned}
$$

where $\hat{\tau}$ is an operator whose characteristic is determined to ensure the (anti-)commutation relations

$$
\begin{aligned}
{\left[\mathfrak{b}_{t}, \tilde{\mathfrak{b}}_{s}\right]_{-\sigma} } & =\left[\mathfrak{b}_{t}, \tilde{\mathfrak{b}}_{s}^{\dagger}\right]_{-\sigma}=0, \\
{\left[\mathfrak{b}_{t}, \mathfrak{b}_{s}^{\dagger}\right]_{-\sigma} } & =\left[\tilde{\mathfrak{b}}_{t}, \tilde{\mathfrak{b}}_{s}^{\dagger}\right]_{-\sigma}=\delta(t-s) .
\end{aligned}
$$

First of all, we require that it should commute with $J_{t}$ and $\tilde{J}_{t}$, and (anti-)commute with $b_{t}$ and $\tilde{b}_{t}$, i.e. $\left[\hat{\tau}, J_{t}\right]=\left[\hat{\tau}, \tilde{J}_{t}\right]=0$ and $\left[\hat{\tau}, b_{t}\right]_{-\sigma}=\left[\hat{\tau}, \tilde{b}_{t}\right]_{-\sigma}=0$. Other properties of the operator $\hat{\tau}$ can be derived using definitions (23). For example, from (23b) one has

$$
\left[\tilde{\mathfrak{b}}_{t}, \tilde{\mathfrak{b}}_{s}^{\dagger}\right]_{-\sigma}=\sigma \hat{\tau}^{2}\left[\tilde{J}_{t} \tilde{b}_{t}, \tilde{b}_{s}^{\dagger} \tilde{J}_{s}\right]_{-\sigma}=\sigma \hat{\tau}^{2} \delta(t-s)
$$

which gives $\hat{\tau}^{2}=\sigma$. On the other hand, commutativity of tilde conjugation and hermitian conjugation, i.e. $\left(\tilde{\mathfrak{b}}_{t}\right)^{\dagger}=\left(\mathfrak{b}_{t}^{\dagger}\right)^{\sim}$, means $\hat{\tau}^{\dagger}=\sigma \hat{\tau}$. From the requirement that $\left(\tilde{\mathfrak{b}}_{t}\right)^{\sim}=\mathfrak{b}_{t}$ and noting that $\left(\tilde{\mathfrak{b}}_{t}\right)^{\sim}=\hat{\tau}(\hat{\tau})^{\sim} J_{t} b_{t}=\hat{\tau}(\hat{\tau})^{\sim} \mathfrak{b}_{t}$, one obtains $(\hat{\tau})^{\sim}=\hat{\tau}^{\dagger}$. 
Thermal degree of freedom can be introduced by the Bogolubov transformation in $\hat{\Gamma}$. For this purpose we require that the expectation value of $\mathfrak{b}_{t}^{\dagger} \mathfrak{b}_{s}$ should be

$$
\left\langle\mathfrak{b}_{t}^{\dagger} \mathfrak{b}_{s}\right\rangle=\bar{n} \delta(t-s)
$$

with $\bar{n} \in \mathbb{R}_{+}$, where $\langle\ldots\rangle=\langle|\ldots|\rangle$ indicates the expectation with respect to tilde invariant thermal vacuums \langle| and |\rangle . The requirement (26) is consistent with the thermal state conditions for states \langle| and |\rangle such that

$$
\begin{aligned}
\langle| \tilde{\mathfrak{b}}_{t}^{\dagger} & =\tau^{*}\langle| \mathfrak{b}_{t}, \\
\tilde{\mathfrak{b}}_{t}|\rangle & =\frac{\tau \bar{n}}{1+\sigma \bar{n}} \mathfrak{b}_{t}^{\dagger}|\rangle, \quad \tau=\sqrt{\sigma} .
\end{aligned}
$$

Let us introduce annihilation and creation operators

$$
\begin{aligned}
\mathfrak{c}_{t} & =[1+\sigma \bar{n}] \mathfrak{b}_{t}-\sigma \tau \bar{n} \tilde{\mathfrak{b}}_{t}^{\dagger}, \\
\tilde{\mathfrak{c}}_{t}^{+} & =\tilde{\mathfrak{b}}_{t}^{\dagger}-\sigma \tau \mathfrak{b}_{t},
\end{aligned}
$$

and their tilde conjugates. From (27) one has \langle| $\mathfrak{c}_{t}^{\text {q }}=\langle| \tilde{\mathfrak{c}}_{t}^{\text {q }}=0$ and $\mathfrak{c}_{t}|\rangle=\tilde{\mathfrak{c}}_{t}|\rangle=0$. With the thermal doublet notations

$$
\begin{aligned}
\overline{\mathfrak{b}}_{t}^{\mu} & =\left(\mathfrak{b}_{t}^{\dagger},-\tau \tilde{\mathfrak{b}}_{t}\right), & & \mathfrak{b}_{t}^{\nu}=\operatorname{collon}\left(\mathfrak{b}_{t}, \tau \tilde{\mathfrak{b}}_{t}^{\dagger}\right), \\
\overline{\mathfrak{c}}_{t}^{\mu} & =\left(\mathfrak{c}_{t}^{+},-\tau \tilde{\mathfrak{c}}_{t}\right), & \mathfrak{c}_{t}^{\nu} & =\operatorname{collon}\left(\mathfrak{c}_{t}, \tau \tilde{\mathfrak{c}}_{t}^{+}\right),
\end{aligned}
$$

(28) and their tilde conjugates can be written in the form of the Bogolubov transformation

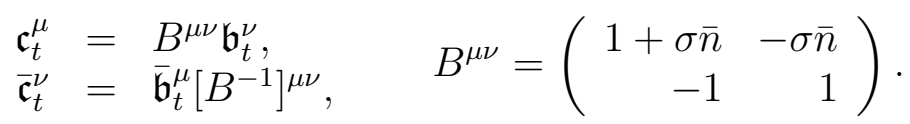

This transformation is canonical since new operators satisfy canonical (anti-)commutation relations

$$
\left[\mathfrak{c}_{t}, \mathfrak{c}_{s}^{+}\right]_{-\sigma}=\delta(t-s) .
$$

In the following, we will use the representation space constructed on vacuums \langle| and |\rangle . Note that $\langle|\neq|\rangle^{\dagger}$, i.e., it is not a unitary representation. Let $\hat{\Gamma}^{\beta}$ denote the Fock space spanned by the basic bra- and ket-vectors introduced by a cyclic operations of $\mathfrak{c}_{t}, \tilde{\mathfrak{c}}_{t}$ on the thermal bra-vacuum \langle| , and of $\mathfrak{c}_{t}^{+}, \tilde{\mathfrak{c}}_{t}^{+}$on the thermal ketvacuum |\rangle . Quantum Brownian motion at finite temperature is defined in the Fock space $\hat{\Gamma}^{\beta}$ by operators

$$
\mathfrak{B}_{t}^{\sharp}=\int_{0}^{t} \mathrm{~d} s \mathfrak{b}_{s}^{\sharp}, \quad \tilde{\mathfrak{B}}_{t}^{\sharp}=\int_{0}^{t} \mathrm{~d} s \tilde{\mathfrak{b}}_{s}^{\sharp},
$$

with $\mathfrak{B}_{0}^{\sharp}=0$ and $\tilde{\mathfrak{B}}_{0}^{\sharp}=0$, where $\sharp$ stands for null or dagger. The explicit representation of processes $\mathfrak{B}_{t}^{\sharp}$ and $\tilde{\mathfrak{B}}_{t}^{\sharp}$ can be performed in terms of the Bogolubov transformation. The couple $\mathfrak{B}_{t}$ and $\mathfrak{B}_{t}^{\dagger}$, for example, is calculated as

$$
\begin{aligned}
& \mathfrak{B}_{t}=\int_{0}^{t} \mathrm{~d} s\left[\mathfrak{c}_{s}+\sigma \tau \bar{n} \tilde{\mathfrak{c}}_{s}^{\text {q}}\right]=\mathfrak{C}_{t}+\sigma \tau \bar{n} \tilde{\mathfrak{C}}_{t}^{\text {q }}, \\
& \mathfrak{B}_{t}^{\dagger}=\int_{0}^{t} \mathrm{~d} s\left[[1+\sigma \bar{n}] \mathfrak{c}_{s}^{\text {q }}+\tau \tilde{\mathfrak{c}}_{s}\right]=[1+\sigma \bar{n}] \mathfrak{C}_{t}^{\text {q }}+\tau \tilde{\mathfrak{C}}_{t},
\end{aligned}
$$


where we defined new operators

$$
\mathfrak{C}_{t}^{\sharp}=\int_{0}^{t} \mathrm{~d} s \mathfrak{c}_{s}^{\sharp}, \quad \tilde{\mathfrak{C}}_{t}^{\sharp}=\int_{0}^{t} \mathrm{~d} s \tilde{\mathfrak{c}}_{s}^{\sharp},
$$

with $\mathfrak{C}_{0}^{\sharp}=0$ and $\tilde{\mathfrak{C}}_{0}^{\sharp}=0$, and $\sharp$ standing for null or the Venus-mark. Since matrix elements of $\mathrm{d} \mathfrak{C}_{t}^{\sharp}$ and $\mathrm{d} \tilde{\mathfrak{C}}_{t}^{\sharp}$ in thermal space $\hat{\Gamma}^{\beta}$ read

$$
\begin{aligned}
& \left\langle\mathrm{d} \mathfrak{C}_{t}\right\rangle=\left\langle\mathrm{d} \tilde{\mathfrak{C}}_{t}\right\rangle=0, \\
& \left\langle\mathrm{~d} \mathfrak{C}_{t}^{+} \mathrm{d} \mathfrak{C}_{t}\right\rangle=\left\langle\mathrm{d} \tilde{\mathfrak{C}}_{t} \mathrm{~d} \tilde{\mathfrak{C}}_{t}^{\text {q }}\right\rangle=0, \\
& \left\langle\mathrm{~d} \mathfrak{C}_{t}^{\text {+ }}\right\rangle=\left\langle\mathrm{d} \tilde{\mathfrak{C}}_{t}^{\text {+ }}\right\rangle=0, \\
& \left\langle\mathrm{~d} \mathfrak{C}_{t} \mathrm{~d} \mathfrak{C}_{t}^{\mathfrak{+}}\right\rangle=\left\langle\mathrm{d} \tilde{\mathfrak{C}}_{t}^{+q} \mathrm{~d} \tilde{\mathfrak{C}}_{t}\right\rangle=\mathrm{d} t,
\end{aligned}
$$

the calculation of moments of quantum Brownian motion in the thermal space $\hat{\Gamma}^{\beta}$ can be performed, for instance, as

$$
\begin{aligned}
\left\langle\mathrm{d} \mathfrak{B}_{t} \mathrm{~d} \mathfrak{B}_{t}^{\dagger}\right\rangle & =\left\langle\left(\mathrm{d} \mathfrak{C}_{t}+\sigma \tau \bar{n} \mathrm{~d} \tilde{\mathfrak{C}}_{t}^{\text {q }}\right)\left([1+\sigma \bar{n}] \mathrm{d} \mathfrak{C}_{t}^{++}+\tau \mathrm{d} \tilde{\mathfrak{C}}_{t}\right)\right\rangle \\
& =[1+\sigma \bar{n}]\left\langle\mathrm{d} \mathfrak{C}_{t} \mathrm{~d} \mathfrak{C}_{t}^{+}\right\rangle \\
& =[1+\sigma \bar{n}] \mathrm{d} t .
\end{aligned}
$$

Repeating this for other pair products of $\mathrm{d} \mathfrak{B}_{t}^{\sharp}, \mathrm{d} \tilde{\mathfrak{B}}_{t}^{\sharp}$ and $\mathrm{d} t$, multiplication rules for these increments can be summarized in the following table:

\begin{tabular}{l|ccccc} 
& $\mathrm{d} \mathfrak{B}_{t}$ & $\mathrm{~d} \mathfrak{B}_{t}^{\dagger}$ & $\mathrm{d} \tilde{\mathfrak{B}}_{t}$ & $\mathrm{~d} \tilde{\mathfrak{B}}_{t}^{\dagger}$ & $\mathrm{d} t$ \\
\hline $\mathrm{d} \mathfrak{B}_{t}$ & 0 & {$[1+\sigma \bar{n}] \mathrm{d} t$} & $\tau \bar{n} \mathrm{~d} t$ & 0 & 0 \\
$\mathrm{~d} \mathfrak{B}_{t}^{\dagger}$ & $\bar{n} \mathrm{~d} t$ & 0 & 0 & $\tau[1+\sigma \bar{n}] \mathrm{d} t$ & 0 \\
$\mathrm{~d} \tilde{\mathfrak{B}}_{t}$ & $\sigma \tau \bar{n} \mathrm{~d} t$ & 0 & 0 & {$[1+\sigma \bar{n}] \mathrm{d} t$} & 0 \\
$\mathrm{~d} \tilde{\mathfrak{B}}_{t}^{\dagger}$ & 0 & $\sigma \tau[1+\sigma \bar{n}] \mathrm{d} t$ & $\bar{n} \mathrm{~d} t$ & 0 & 0 \\
$\mathrm{~d} t$ & 0 & 0 & 0 & 0 & 0
\end{tabular}

As far as we know, the result (38) is the first report about quantum boson and fermion stochastic calculus valid for the stationary non-equilibrium case. Similar result for a purely boson system was obtained in [39]. In [40] we derive stochastic calculus valid for both stationary and non-stationary cases and use them for the analysis of quantum stochastic differential equations. The obtained equations include quantum Langevin equation and quantum stochastic Liouville equation together with the corresponding master equation. The Fokker-Planck equation is derived by taking the random average of the corresponding stochastic Liouville equation. The relation between the Langevin equation and the stochastic Liouville equation, as well as between the Heisenberg equation for the operators of gross variables and the Fokker-Planck equation obtained there, is similar to the one between the Heisenberg equation and the Schrödinger equation in quantum mechanics and field theory. The application of quantum stochastic differential equations in particular problems will be presented elsewhere. 


\section{References}

1. Chandrasekhar S. // Rev. Mod. Phys., 1943, vol. 15, p. 1.

2. Wang M.C., Uhlenbeck G.E. // Rev. Mod. Phys., 1945, vol. 17, p. 323.

3. Mazo R.T. Browniam Motion. Fluctuations, Dynamics and Applications. Oxford, Clarendon Press, 2002.

4. Gardiner C.W. Handbook of Stochastic Methods for Physics, Chemistry and Natural Sciences. 2nd edn. Berlin, Springer-Verlag, 1985.

5. Itô K. // Proc. Imp. Acad. Tokyo, 1944, vol. 20, p. 519.

6. Stratonovich R. // J. SIAM Control, 1966, vol. 4, p. 362.

7. Stochastic Processes in Chemical Physics (Ed. Shuler K.E.). - In series: Advances in Chemical Physics, vol. XV (Eds. Prigogine I., S. Rice). New York, Interscience Publishers, 1969.

8. Coffey W. Development and application of the theory of Brownian motion. - In: Dynamical Processes in Condensed Matter (Ed. by Evans M.W.), p. 69-252. - In series: Advances in Chemical Physics, vol. LXIII (Ed. by Prigogine I., Rice S.). New York, Interscience Publishers, 1985.

9. Sobczyk K. Stochastic Differential Equations: with Application to Physics and Engineering. Dordrecht, Kluwer Academic, 1991.

10. Van Kampen N.G. Stochastic Processes in Physics and Chemistry. Amsterdam, North Holland, 1992.

11. Coffey W.T., Kalmykov Yu.P., Waldron J.T. The Langevin Equation. With Applications in Physics, Chemistry and Electrical Engineering. Singapore, World Scientific, 1996.

12. Anderson P.W. // J. Phys. Soc. Japan, 1954, vol. 9, p. 316.

13. Kubo R. // J. Phys. Soc. Japan, 1954, vol. 9, p. 935.

14. Kubo R. A stochastic theory of line-shape and relaxation. - In: Fluctuation, Relaxation and Resonance in Magnetic Systems. Edinburgh-London, Oliver and Boyd, 1962, p. 23-68.

15. Kubo R. // J. Math. Phys., 1963, vol. 4, p. 174.

16. Senitzky I.R. // Phys. Rev., 1960, vol. 119, p. 670; 1961, vol. 124, p. 642; 1963, vol. 131, p. 2827.

17. Schwinger J. // J. Math. Phys., 1961, vol. 2, p. 407.

18. Haken H. // Z. Phys., 1964, vol. 181, p. 96; 1965, vol. 182, p. 346.

19. Haken H. Optik. Handbuch der Physik, vol. XXV/2c. Berlin, Springer-Verlag, 1970.

Reprinted as: Haken H. Laser Theory. Berlin, Springer-Verlag, 1984.

20. Haken H. // Rev. Mod. Phys., 1975, vol. 47, p. 67.

21. Lax M. // Phys. Rev., 1966, vol. 145, p. 110.

22. Kubo R. // J. Phys. Soc. Japan Suppl., 1969, vol. 26, p. 1.

23. Davies E.B. // Commun. Math. Phys., 1969, vol. 15, p. 227.

24. Davies E.B. Quantum Theory of Open Systems. London, Academic Press, 1976.

25. Cockroft A.M., Hudson R.L. // J. Multivar. Anal., 1977, vol. 7, p. 107.

26. Hudson R.L., Streater R.F. // Phys. Lett. A, 1981, vol. 86, p. 277.

27. Hudson R.L., Parthasarathy K.R. // Commun. Math. Phys., 1984, vol. 93, p. 301; Acta Appl. Math., 1984, vol. 2, p. 353; Hudson R.L., Lindsay J.M. // J. Func. Anal., 1985, vol. 61, p. 202.

28. Accardi L., Frigerio A., Lewis J.T. // Publ. RIMS (Kyoto), 1982, vol. 18, p. 97; 
Accardi L. // Rev. Math. Phys., 1990, vol. 2, p. 127.

29. Accardi L., Lu Y.G., Volovich I. Quantum Theory and its Stochastic Limit. Berlin, Springer, 2002.

30. Parthasarathy K.R. // Pramãna - J. Phys., 1985, vol. 25, p. 457; Rev. Math. Phys., 1989, vol. 1, p. 89.

31. Parthasarathy K.R. An Introduction to Quantum Stochastic Calculus. - In series: Monographs in Mathematics, vol. 85. Basel, Birkhäuser, 1992.

32. Applebaum D., Hudson R.L. // Commun. Math. Phys., 1984, vol. 96, p. 473; J. Math. Phys., 1984, vol. 25, p. 858.

33. Applebaum D. // J. Phys. A, 1986, vol. 19, p. 937; 1995, vol. 28, p. 257.

34. Hudson R.L., Parthasarathy K.R. // Commun. Math. Phys., 1986, vol. 104, p. 457.

35. Parthasarathy K.R., K.B. Sinha // Pramãna - J. Phys., 1986, vol. 27, p. 105.

36. Barnett C., Streater R.F., Wilde I.F. // J. Funct. Anal., 1982, vol. 48, p. 172; J. London Math. Soc., 1983, vol. 27, p. 373; Commun. Math. Phys., 1983, vol. 89, p. 13; J. Func. Anal., 1983, vol. 52, p. 19.

37. Arimitsu T., Umezawa H. // Progr. Theor. Phys., 1985, vol. 74, p. 429; 1987, vol. 77, p. $32 ; 1987$, vol. 77 , p. 53.

38. Arimitsu T. // Condens. Matter. Phys., 1994, vol. 4, p. 26.

39. Saito T., Arimitsu T. // J. Phys. A, 1997, vol. 30, p. 7573.

40. Kobryn A.E., Hayashi T., Arimitsu T. // Ann. Phys., 2003, vol. 308, p. 395. 
Квантове стохастичне числення: бозонний та ферміоний броунівський рух

\author{
O.Є.Кобрин ${ }^{1,2}$, Ц.Хаяші ${ }^{1}$, Т.Аріміцу ${ }^{1}$
}

1 Інститут фізики Університету м. Цукуба,

Японія, префектура Ібаракі 305-8571

2 Інститут молекулярних досліджень, Міодаіджі, Японія, м. Оказакі, префектура Аічі 444-8585

Отримано 5 вересня 2003 р.

Динаміка квантових систем, що зазнають збурення через лінійну взаємодію із термостатом стохастично, може бути описана за допомогою квантових стохастичних диференціальних рівнянь (наприклад, квантового стохастичного рівняння Ліувіля, або квантового рівняння Ланжевена). Для цього необхідно дати означення квантового броунівського руху. Оскільки донедавна воно було наведене лише для бозонних систем, у даній роботі показується як таке означення можна ввести у випадку ферміонних систем.

Ключові слова: стохастичні процеси, бозонний броунівський рух, ферміонний броунівський рух

PACS: $02.50 . E y, 05.30 .-d$ 